\title{
Actividades de enfermería profesional en los equipos de primer nivel de atención de Uruguay
}

\author{
Estela Harispe* ${ }^{\star}$ Fiorella Cavalleri†, Ima León
}

\section{Resumen}

Objetivo: determinar cuáles son las actividades que deberían desarrollar las enfermeras profesionales en el primer nivel de atención (PNA), en el marco de la estrategia de atención primaria en salud (APS).

Método: estudio exploratorio de consulta a expertos de la academia, en dos etapas.

Resultados: participaron nueve expertas de enfermería comunitaria de la Facultad de Enfermería de la Universidad de la República Oriental del Uruguay. Los principales resultados consistieron en la obtención de un consenso de expertos de la academia, sobre las actividades que debe desarrollar la enfermería profesional en el PNA.

Conclusiones: la amplitud de las actividades que las enfermeras profesionales pueden realizar en el PNA y la incorporación del marco conceptual de la A reafirma la importancia de esta profesión como integrante del equipo de salud para impulsar el cambio de modelo de atención propuesto por el Sistema Nacional Integrado de Salud (SNIS).

Establecer las actividades de la profesión de enfermería en el PNA permitirá definir mejor su rol en el equipo de salud y potenciar su desarrollo. Es importante conocer los resultados de la investigación de actividades de los médicos de familia, las opiniones que tiene cada grupo respecto de ambas profesiones y continuar investigando el desarrollo complementario, suplementario o sustituto de las actividades de estas y otras profesiones del equipo de salud.

Palabras clave: Enfermería

Uruguay

Enfermería de atención primaria

Key words: $\quad$ Nursing

Uruguay

Primary care nursing

\footnotetext{
* Egresada de la Maestría en Gerencia y Administración con concentración en Servicios de Salud de la Facultad de Ciencias Económicas y Administración. UDELAR.

† Prof. Adj. Dpto. de Medicina Preventiva y Social. Facultad de Medicina. UDELAR.

$\ddagger$ Prof. Agda. Dpto. de Medicina Preventiva y Social. Facultad de Medicina. UDELAR.

Correspondencia: Lic. Estela Harispe. Correo electrónico: eharispe@gmail.com

Recibido: 7/6/19

Aprobado: 9/9/19
} 


\section{Introducción}

A partir del año 2005, Uruguay comenzó un proceso transformador del sistema de salud cuyo objetivo fue avanzar hacia la universalización de la protección social en materia de salud a toda la población. Dicho sistema propuso cambios en su modelo de financiamiento, gestión y atención ${ }^{(1)}$.

El cambio de modelo de atención tuvo como una de sus bases fundamentales (según lo establece la Ley $18211)^{(2)}$, la estrategia de atención primaria en salud (APS) y la priorización del primer nivel de atención (PNA). En este nivel, el abordaje del proceso salud enfermedad tiene una estrecha relación con aspectos sociales y culturales de las personas, familias y comunidades. Exige, por lo tanto, competencias disciplinares específicas del área social y de salud pública. El trabajo en equipo y con redes institucionales y organizaciones es ineludible y requiere capacidades de relación, negociación y articulación. La complejidad organizativa es alta e implica el uso de estrategias que permitan resolver con eficiencia la gestión de recursos distribuidos en un amplio territorio. Los profesionales de la salud tienen integrada dentro de su formación académica la adquisición de conocimientos y herramientas para realizar estos abordajes, sin ser exclusivos de una profesión. Esto puede dificultar la asignación de roles en el equipo a unos u otros.

Existen diferentes opiniones en cuanto a cómo deben componerse los equipos de PNA, pero hay dos profesionales que están siempre presentes: el médico y la enfermera $^{(3,4)}$. Si el médico debe estar especializado o no y la enfermera ser profesional o auxiliar, son debates que siguen vigentes. Se ensayan modelos diferentes de acuerdo a las realidades epidemiológicas, socioculturales y a la oferta y perfil profesional de los integrantes del equipo de cada país. Según la Organización Panamericana de la Salud (OPS): "No hay, pues, uniformidad en cuanto a la composición de los equipos de APS en el primer nivel de atención, si bien en general se considera una composición mínima a partir de médico (general, de familia, etc.), enfermera y un técnico de nivel medio con funciones de auxiliar, o de técnico comunitario, en función de las necesidades de la comunidad"(4).

Las actividades pueden ser realizadas por distintas disciplinas y es conveniente para su abordaje el trabajo interdisciplinar en el marco del equipo de salud ${ }^{(4,5)}$. Se propone que sea el propio equipo el que distribuya las actividades entre sus integrantes de acuerdo a sus competencias específicas ${ }^{(6)}$. Esto somete a los profesionales a tensiones; en la práctica, la construcción de un equipo de trabajo es un desafío importante y requiere un esfuerzo dirigido. Según la $\mathrm{OPS}^{(4)}$, el trabajo en equipo "no se logra mediante la mera yuxtaposición física de sus componentes y actividades; es necesario que asuman objeti- vos comunes y que se establezcan entre ellos vínculos funcionales que posibiliten un desarrollo armónico y un conjunto de tareas, basándose en la división funcional del trabajo y de las responsabilidades compartidas de acuerdo a la capacitación técnica de los profesionales de salud que lo integran, en lugar de basarse en una línea jerárquica vertical".

La construcción de equipos requiere el reconocimiento de los aportes de todos sus integrantes. Este reconocimiento y delimitación disciplinar no está exento de tensiones y contradicciones. El "saber", como espacio de poder, está presente tanto en el ámbito académico como en el profesional. Muy pocos estudios exploran cómo se reconocen los aportes disciplinares y asignan actividades, lo que dificulta desarrollar políticas de formación y modelos adecuados de dotación de recursos humanos ${ }^{(7)}$.

A nivel nacional, pocos trabajos han abordado el tema de la composición de los equipos de PNA ${ }^{(6,8,9)}$. En ningún caso se ha propuesto cuáles deben ser las actividades de cada profesional del equipo.

La composición del equipo es fundamental en los sistemas de salud, tanto por su impacto en la calidad de la atención como por los costos que genera ${ }^{(10)}$.

Las actividades que se asignan a una profesión, su rol social, su desarrollo y las asociaciones con otras profesiones devienen de un complejo proceso sociopolítico con múltiples actores ${ }^{(7,11,12)}$.

Uruguay ha presentado históricamente una dotación de recursos humanos en salud privilegiados en relación con América Latina y una dotación de médicos por habitantes superior al promedio de Europa ${ }^{(13)}$. En el año 2017 había registrados 16.268 médicos, 6.176 licenciados en enfermería y 23.606 auxiliares de enfermería ${ }^{(10)}$, para una población de 3.440 .157 habitantes ${ }^{(14)}$. Sin embargo, estas cifras denotan una dotación relativa de médicos y auxiliares de enfermería elevada para la dotación de enfermeras profesionales, lo que preocupa a la autoridad sanitaria. En términos generales existe una relación de 1,5 auxiliares de enfermería por cada médico y 2,6 médicos por cada licenciada en enfermería. Las enfermeras profesionales concentran su actividad en el segundo y tercer nivel de atención, donde la oferta laboral es mayor. Esta composición de los recursos humanos en salud (RHS) ha sido reconocida por los actores sanitarios como una dificultad para el avance del cambio de modelo de atención debido a que las competencias de las enfermeras profesionales son fundamentales para avanzar en el desarrollo de la estrategia de APS $^{(10)}$. El rol asignado a la licenciada en enfermería resultante de esta composición del equipo tiene un fuerte componente en gestión. Esto se acompaña de una mayor elección de formación de posgrado en el área de gestión de servicios 
por parte de las profesionales ${ }^{(15)}$. El tiempo dedicado a cuidados directos es escaso, lo que puede incidir en su calidad $^{(16)}$. A diferencia de varios países desarrolla$\operatorname{dos}^{(17,18)}$, las actividades independientes como consulta y derivación son aún incipientes, la prescripción de medicamentos no integra sus actividades ni está reconocida legalmente.

La enfermería profesional está avanzando en el marco legal de competencias, logrando la promulgación de la Ley 18.815, Profesión de Enfermaría de $2011^{(19)}$ y su posterior reglamentación por el Poder Ejecutivo ${ }^{(20)}$. Se le asignan amplias actividades vinculadas al cuidado de la salud de los individuos, familias y comunidades, a la gestión de servicios y programas, a la docencia e investigación. Solo en tres de las 53 competencias definidas en el decreto, hay una vinculación al cumplimiento de la indicación médica.

La Facultad de Enfermería ha desarrollado cursos de posgrado, la especialidad y la Maestría en Enfermería Comunitaria $^{(21,22)}$. En el año 2018, recibió la acreditación de calidad académica MERCOSUR por el sistema ACRU- SUR para la carrera de Enfermería de la Universidad de la República Oriental del Uruguay (UDELAR) $)^{(23)}$.

La definición de las actividades que pueden o deben realizar las enfermeras profesionales en el PNA en Uruguay es imprescindible para determinar la composición de los equipos de atención del primer nivel y cuantificar las necesidades de formación de estas profesionales.

La investigación consistió en un estudio exploratorio basado en consulta a expertos en tres etapas. Se buscó determinar la opinión de la academia en relación con las actividades que deberían desarrollar los médicos de familia y comunidad y las enfermeras profesionales en el PNA en Uruguay.

Se trabajó con expertos de la UDELAR por ser la formadora de más del $90 \%$ de los egresados de Medicina y Enfermería profesional en el país ${ }^{(24)}$.

En este artículo se presentan los resultados de la primera y segunda etapa respecto al grupo de expertos de enfermería. $\mathrm{Su}$ objetivo fue determinar las actividades que deben desarrollar las enfermeras profesionales en el PNA, en el marco de la estrategia de atención primaria desde la perspectiva del Departamento de Enfermería Comunitaria de la UDELAR.

\section{Material y método}

Se realizó una investigación de tipo exploratorio y descriptivo. Se propuso una estrategia de consenso de expertos a efectos de conocer la perspectiva de la academia sobre el objeto de estudio.

El universo de estudio estuvo constituido por un profesor (grado 5), cuatro profesores agregados (grado 4) y siete profesores adjuntos (grado 3) del Departamento de Enfermería Comunitaria de la UDELAR.

El período de consulta a los expertos fue de enero a octubre de 2017.

\section{Variables de estudio}

Funciones/actividades* de enfermería en el PNA.

\section{Etapa I. Conformación del listado de actividades}

Se propuso obtener un listado de actividades que deben desarrollar las enfermeras profesionales en el PNA de Uruguay en el marco de la estrategia de atención primaria.

Para esta primera etapa se generó un cuestionario semiestructurado con la suficiente amplitud para recoger las opiniones de los expertos en distintas áreas de la APS.

El formulario se diseñó a partir de 15 funciones de los equipos de $\mathrm{PNA}^{(4,6)}$ y se estructuró con preguntas semiabiertas en las que cada experto debía definir y fundamentar hasta cinco actividades de su profesión para cada una de las 15 funciones del equipo.

Se envió a cada experto vía correo electrónico.

Las respuestas se agruparon por función en una planilla única, obteniendo un listado de actividades por función.

Posteriormente se realizó:

- Identificación de respuestas con orientación a un mismo tema, conformando así varias categorías** de actividades para cada función.

- Reagrupación de las respuestas según esas categorías. Las respuestas podrían incluirse en más de una categoría si se consideraba necesario, ya que algunas contenían varias actividades en la misma oración.

- Redacción de actividades consolidadas incorporando las similares e incluibles en una redacción única.

En todos los casos se buscó cuidar la esencia de la respuesta de las expertas.

\section{Etapa II. Búsqueda de consensos}

El objetivo de esta etapa fue conformar un listado de actividades consensuadas y no consensuadas por las expertas.

\footnotetext{
* En el cuestionario se usaron estos dos términos como sinónimo, ya que su uso es similar como puede observarse en las siguientes definiciones de la Real Academia Española:

Función: tarea que corresponde realizar a una institución o entidad, o a sus órganos o personas.

Actividad: conjunto de operaciones o tareas propias de una persona o entidad.

En el artículo usaremos siempre el término actividad/es cuando nos referimos a cada profesión y funciones cuando nos referimos al equipo. ** Definición: cada una de las clases o divisiones establecidas al clasificar algo (Real Academia Española)
} 
Se realizaron dos rondas de preguntas vía correo electrónico.

Para la primera ronda se diseñó un formulario con las actividades consolidadas a partir de las respuestas obtenidas en la primera etapa. El formulario se envió a cada experta.

En esta etapa se pusieron a consideración 152 actividades.

Con las actividades que no reunieron consenso o tenían observaciones de forma se generó un nuevo listado para ser evaluadas por las expertas. En los casos de observaciones sobre aspectos de forma se propuso un cambio en la redacción de la actividad consolidada. Se consignaron las fundamentaciones discrepantes manteniendo el anonimato.

El nuevo formulario se envió a cada experta y se solicitó manifestar su acuerdo (Sí) o desacuerdo (No) fundamentando las respuestas.

Con las respuestas se conformó un listado indicando las actividades consensuadas y no consensuadas con los principales aspectos en discusión. Posteriormente se realizó una redacción final de las actividades y una recategorización, suprimiendo los aspectos redundantes.

\section{Resultados}

Participaron en el estudio nueve de las 12 expertas invitadas. Tres profesoras agregadas (grado 4) y seis profesoras adjuntas (grado 3).

En la primera etapa se obtuvieron 538 respuestas de actividades (de las 675 posibles) y 175 fundamentos.

Las respuestas se consolidaron en 152 actividades de las cuales 136 obtuvieron consenso en incluirlas como actividades propias de la profesión de enfermería; 16 actividades no fueron consensuadas.

Las 15 funciones iniciales se recategorizaron en siete, permitiendo eliminar las actividades redundantes y obteniendo finalmente 69 actividades consensuadas. Se presenta en la tabla 1 una síntesis de las actividades que se consideraron relevantes para la publicación.

Los disensos fueron escasos y no generaron intercambios conceptuales destacables entre las expertas.

Estuvieron centrados en:

- La delimitación del área independiente de enfermería.

- La participación en instituciones gremiales como actividad profesional.

- La participación en comisiones de seguridad del paciente.

- Delimitación del ámbito operativo y de gestión: no logró consensuar actividades como gestión de agendas, gestión de recursos compartidos por varias instituciones y estudios de costos.
Tabla 1. Funciones y actividades de enfermería en el primer nivel de atención.

\section{Categoría 1. Diagnóstico de Salud y Planificación}

Planificar, organizar y actualizar el análisis de situación de salud participativo (ASSP), estimulando la participación de todo el equipo y la población.

Realizar el relevamiento de datos y participar en el procesamiento, análisis e interpretación, elaborando conclusiones y sugerencias.

Identificar los determinantes de salud del territorio y los datos epidemiológicos de la población y mapearlos.

Definir un plan de trabajo incluyendo las necesidades de recursos para su implementación y establecer indicadores, mecanismos de registro y control que permitan monitorear y evaluar su cumplimiento.

Participar y asesorar en la planificación sanitaria a nivel comunal y/o municipal y expresar en los ámbitos que correspondan las demandas de la población y del equipo para contribuir a la mejora de las condiciones de vida.

Categoría 2. Proceso asistencial a individuos, familias y comunidades

Coordinar y realizar actividades tendientes a la promoción de la salud y generación de entornos saludables.

Realizar acciones de prevención en los distintos grupos etarios y grupos de riesgo.

Participar en la búsqueda activa de individuos, familias y grupos con dificultades en el acceso.

Realizar la consulta de enfermería y visita domiciliaria (CE/VD) con enfoque familiar, elaborando un plan de cuidados de enfermería (PAE) que se retroalimente de acuerdo a la evolución de la situación de salud.

Realizar y/o delegar y supervisar, técnicas y procedimientos de enfermería derivadas del proceso de atención de enfermería o de la prescripción médica.

Incentivar y colaborar en el eficaz control con su médico de referencia.

Educar a la familia y/o cuidador/a principal en aspectos del cuidado de la persona que recibe cuidados paliativos, brindar apoyo y contención.

Evaluar las acciones en las que interviene la profesional y/o el equipo de enfermería, utilizando la información para retroalimentar futuras actividades.

Categoría 3. Organización y coordinación del proceso asistencial

Propiciar mejoras en la organización del servicio, ampliar horarios y articular servicios tomando como centro al usuario y realizar acciones para que familias y comunidades identifiquen el servicio de salud de primer nivel como puerta de entrada para su atención.

Gestionar los programas/planes de atención de las personas del área de responsabilidad en los distintos grupos etarios a lo largo de la vida.

Desarrollar mecanismos de coordinación (enfermería de enlace, seguimiento domiciliario, etc.) y contribuir a la gestión y/o coordinación de una referencia y contrarreferencia efectiva.

Diseñar, proponer y ejecutar y evaluar los programas de educación para la salud en todos los ámbitos del accionar de enfermería.

Diseñar, elaborar, ejecutar y evaluar manuales, protocolos y guías de cuidados de enfermería en el primer nivel de atención (PNA).

Derivar al/los profesionales que corresponda según el análisis de la situación de salud-enfermedad.

(continúa en la página siguiente) 
(viene de la página siguiente)

Categoría 4. Gestión de recursos

Promover el trabajo en Redes Integradas de salud (RISS) optimizando las respuestas a la realidad y necesidades de cada zona.

Gestionar el equipo de enfermería, determinando y procurando necesidades de recursos humanos y delegando las actividades pertinentes a cada situación.

Contribuir al trabajo en equipo y a su motivación promoviendo el accionar complementario y coordinado.

Investigar la cantidad y frecuencia de utilización ideal de recursos.

Gestionar la obtención de recursos materiales y equipos necesarios e incentivar su buen uso y mantenimiento estableciendo protocolos de utilización y mecanismos de control que aseguren un uso eficaz y eficiente.

Coordinar la utilización de recursos compartidos por varios servicios, sectores e instituciones, favoreciendo su optimización y evitando duplicaciones.

Contribuir en la adecuada gestión de los residuos, comunes y sanitarios.

Velar por cumplimiento de las normas relativas a las condiciones y medioambiente laboral de enfermería.

Categoría 5. Coordinación intersectorial y participación comunitaria

Estimular la participación de la comunidad como generadora de nuevos conocimientos y promover su capacitación en derechos.

Promover una buena comunicación del equipo con la población, dando respuesta a sus necesidades, con un trato amigable e informando sobre la mejor utilización de los servicios.

Realizar coordinaciones intersectoriales y alianzas con múltiples actores de la comunidad desarrollando actividades de promoción, prevención, salud laboral, etc., definiendo en cada caso los roles de cada organismo e institución.

Sistematizar y evaluar acciones interinstitucionales e intersectoriales.

\section{Categoría 6. Docencia}

Identificar las necesidades e intereses de capacitación del equipo de salud, planificar, coordinar y participar en actividades incidentales y programadas de educación para todo el equipo de salud, promoviendo el aporte de cada disciplina en el proceso educativo.

Promover y participar activamente en las actividades de formación de grado y posgrado en el servicio, con estudiantes de todas las profesiones del equipo de salud.

Participar en cursos de actualización, especialidades, maestrías y doctorados para mejorar la formación profesional.

\section{Categoría 7. Investigación (seis actividades)}

Investigar y monitorear problemas de salud con enfoque en determinantes sociales y promover el interés del equipo por la investigación y búsqueda de evidencias científicas.

Impulsar y participar activamente en las propuestas, elaboración y ejecución de proyectos que generen nuevos conocimientos.

Promover la participación activa de enfermería en los ámbitos científicos.

Divulgar los resultados de las investigaciones en publicaciones, congresos, jornadas, etc.

\section{Discusión}

Se observó que las expertas comparten el marco conceptual de la PPS $^{(3)}$. Plantearon la importancia de desarrollar un primer nivel que atienda las necesidades de la población, resolutivo, que brinde una atención integral, integrada y longitudinal, con un enfoque familiar y comunitario. Se identificaron como asesoras en la planificación sanitaria y como representantes del equipo y de los usuarios para ciertos ámbitos. En la mayoría de las actividades se destacó la importancia del trabajo en equipo, la participación e involucramiento de la población, el trabajo interinstitucional e intersectorial para el abordaje de los determinantes sociales y la complementación de recursos. Incluyeron actividades de capacitación e investigación contemplando la realidad local y las necesidades de los equipos y la población.

En la función asistencial las enfermeras delimitaron su ámbito de trabajo diferenciando áreas independientes y otras dependientes de la actividad de los médicos. Desarrollaron con mayor amplitud las independientes de su profesión, dentro de las cuales enfatizaron en la consulta de enfermería, la visita domiciliaria y la derivación, haciendo referencia al proceso de atención de enfermería (PAE) y al diagnóstico de enfermería. Consensuaron la asignación de estas actividades a la profesión, lo que es coincidente con las competencias establecidas en la ley de enfermería. Sin embargo, en Uruguay estas prácticas, a diferencia de otros países ${ }^{(18)}$, son aún incipientes. Recientemente, se incluyó la consulta de enfermería como práctica independiente en el mayor prestador público del país, la Administración de los Servicios de Salud del Estado. Esto posibilita la agenda directa del usuario con la profesional y el registro en la historia clínica electrónica ${ }^{(25)}$.

Hubo escasa discusión y desarrollo de elementos conceptuales o metodológicos de la atención de enfermería. No se explicitaron aspectos vinculados a la relación enfermera/paciente. Respecto a la gestión de casos, no se logró consenso e impresiona no haber un desarrollo teórico suficiente.

Se identificó la relevancia de la protocolización de las acciones y de la necesidad de trabajar con programas. La amplitud de las actividades de gestión y coordinación vinculadas al proceso administrativo, planificación, organización, supervisión/control y evaluación denotan un importante desarrollo de esta función. Se visualizaron planificando los servicios, gestionando los recursos materiales y coordinando las acciones con otros servicios y actores. Incluyeron elementos conceptuales y metodológicos diversos. En estas áreas (coordinación y gestión), mencionaron como su campo de acción el servicio en general. Cuando se refirieron a la gestión de recursos humanos, sin embargo, especificaron que su campo de acción es el equipo de enfermería. Con los de- 
más trabajadores se identificaron en un rol coordinador, no jerárquico. Este desarrollo es coincidente con su perfil de competencias profesionales y con el rol asignado predominantemente a la profesión en Uruguay ${ }^{(16,20,22)}$.

\section{Conclusiones}

Las actividades que las enfermeras profesionales pueden realizar en el PNA y la incorporación del marco conceptual de la APS reafirma la importancia de esta profesión como integrante del equipo de salud para impulsar el cambio de modelo de atención propuesto por el SNIS.

El desarrollo de políticas que estimulen una mayor dotación de estas profesionales posibilitará la asignación en la práctica de actividades que ya están reglamentadas y reconocidas como propias por la academia. La realización por parte de las enfermeras profesionales de consulta de enfermería, visitas domiciliarias, derivación y coordinación de la atención podría mejorar la función de puerta de entrada al sistema, otorgando una mayor accesibilidad y continuidad del proceso de atención.

Establecer las actividades de la profesión de enfermería en el PNA permitirá definir mejor su rol en el equipo de salud y potenciar su desarrollo.

Es importante conocer los resultados de la investigación de actividades de los médicos de familia, las opiniones que tiene cada grupo respecto de ambas profesiones y continuar investigando el desarrollo complementario, suplementario o sustituto de las actividades de estas y otras profesiones del equipo de salud.

\section{Abstract}

Objective: to define the activities that should be conducted by professional nurses of the primary healthcare level (PHC) within the framework of the Primary Health Care Strategy.

Method: exploratory study based on consultation to academic experts and scholars in two stages.

Results: nine Community Nursing Experts from the School of Nursing of the University of the Republic of Uruguay participated in the study. The main result was the achievement of a consensus by academic experts on the activities to be developed by professional nurses in the primary healthcare level.

Conclusions: the wide array of activities that may be developed by nurses in the Primary Healthcare Level and the inclusion of the Primary Health Care Strategy conceptual framework ratifies the importance of these professionals as members of the health team to encourage change in the healthcare model proposed by the $\mathrm{Na}$ tional Integrated Health System.

Defining the activities to be developed by nursing professionalS in the primary healthcare level will enable a more accurate description of their role in the health team and maximise their development. It is important to learn about the results on activities conducted by general practitioners and the opinions of each group with regard to both professions, and to continue to study the development of complementary, supplementary or substitute activities by these and other professionals in the health team.

\section{Resumo}

Objetivo: determinar quais são as atividades que deveriam ser desenvolvidas pelas enfermeiras universitárias no Primeiro Nível de Atenção (PNA), no âmbito da estratégia de Atenção Primária de Saúde (APS).

Método: estudo exploratório de consulta a peritos acadêmicos, em duas etapas.

Resultados: participaram nove peritos de Enfermería Comunitária da Facultad de Enfermería da Universidad de la República Oriental del Uruguay. Os principais resultados, consistiram na obtenção de um consenso de peritos acadêmicos sobre as atividades que deve ser desenvolvidas pelas enfermeiras universitárias no PNA.

Conclusões: a amplitude das atividades que as enfermeiras universitárias podem realizar no PNA e a incorporação dos conceitos da APS, reafirma a importância desta profíssão como integrante da equipe de saúde para promover a mudança de modelo de atenção proposto pelo Sistema Nacional Integrado de Salud (SNIS).

Estabelecer as atividades da profissão das enfermeiras universitárias no PNA permitirá definir melhor seu papel na equipe de saúde e potenciar seu desenvolvimento. É importante conhecer os resultados da pesquisa de atividades dos médicos de família, as opiniões que cada grupo tem a respeito de ambas profissões e continuar investigando o desenvolvimento complementar, suplementar ou substitutivo das atividades destas e de outras profissões da equipe de saúde.

\section{Bibliografía}

1. Uruguay. Ministerio de Salud Pública. Transformar el futuro: metas cumplidas y desafíos renovados en el Sistema Nacional Integrado de Salud. Montevideo: MSP, 2011. Disponible en: http://www.msp.gub.uy/sites/default/files/archivos_adjuntos/MSP_libro_reforma.pdf [Consulta: 16 febrero 2018].

2. Ley 18211. Creación del Sistema Nacional Integrado de Salud. Montevideo, 13 de diciembre de 2007. Disponible en https://www.impo.com.uy/bases/leyes/18211-2007.[Consulta: 16 febrero 2018].

3. Organización Panamericana de la Salud. La Renovación de la atención primaria de salud en las Américas: documento de posición de la Organización Panamericana de la Sa- 
lud/Organización Mundial de la Salud (OPS/OMS). Washington, DC: OPS, 2007.

4. Organización Panamericana de la Salud. Los equipos de APS. En: Sistemas de salud basados en la atención primaria de salud: estrategias para el desarrollo de los equipos de APS. Washington, D.C.: OPS, 2008:10-2. (La renovación de la Atención Primaria de Salud en las Américas, 1).

5. Organización Panamericana de la Salud, División de Desarrollo de Sistemas y Servicios de Salud (HSP), Programa de Organización y Gestión de Sistemas y Servicios de Salud (HSO). La enfermería de salud pública y las funciones esenciales de salud pública: bases para el ejercicio profesional. Rev Enferm IMSS 2004; 12(3):159-170. Disponible en: https://www.medigraphic.com/pdfs/enfermeriaimss/eim-2004/eim043g.pdf [Consulta: 21 junio 2018].

6. Uruguay. Ministerio de Salud Pública. División Recursos Humanos del SNIS. Equipos de salud del Primer Nivel de Atención (DT 01/14). Montevideo: MSP, 2014.

7. Rovere MR. Planificación estratégica de recursos humanos en salud. Washington, DC: OPS, 1993. (Desarrollo de recursos humanos en salud, 96).

8. Uruguay. Ministerio de Salud Pública. Reestructura del Primer Nivel de Atención. Mercado de trabajo del sector salud.(Documentos de trabajo de Economía de la Salud n³/06). Montevideo: MSP, 2006.

9. Rodriguez V, Barrios A. El equipo de Salud en el ámbito comunitario. Propuesta de discusión en el marco del proceso de Regionalización. Montevideo: Facultad de Medicina. Departamento de Medicina General, Familiar y Comunitaria, 2007.[Monografía de grado].

10. Uruguay. Ministerio de Salud Pública. A diez años de iniciada la reforma sanitaria: evaluación y desafíos del Sistema Nacional Integrado de Salud. (Jornadas de Intercambio). Montevideo: MSP, 2017.

11. Fernández Pérez JA. Estructura y formación profesional. El caso de la profesión médica. Puebla: Facultad de Filosofía y Letras. Benemérita Universidad Autónoma de Puebla, 2002.

12. Freidson E. La profesión médica. Un estudio de sociología del conocimiento aplicado. Barcelona: Península, 1978.

13. Nuñez S. Los desafíos de Toronto medición de las metas regionales de recursos humanos para la salud 2007 - 2015 Uruguay (DT $\mathrm{N}^{\mathrm{o}}$ 01/13). 2013. Disponible en: https://www.paho.org/uru/index.php?option=com_docman\&view $=$ download\&alias $=406$-metas-regionales-de-rrhh-div-rhs-del-snis\&category_slug=publications\&Itemid $=307$ [Consulta: 20 agosto 2017].

14. Uruguay. Instituto Nacional de Estadística. Uruguay en cifras 2014. Disponible en: http: //www.ine.gub.uy/web/guest/ uruguay-en-cifras. [Consulta: 27 agosto 2017].
15. Nuñez S, Benia W, coords. Enfermería en el Uruguay. Montevideo: OPS, 2015

16. Uruguay. Ministerio de Salud Pública. División de Recursos Humanos del SNIS. Dotación y condiciones de trabajo de enfermería en el Uruguay (DT N ${ }^{\circ} / 12$ ). Disponible en: https://www.gub.uy/ministerio-salud-publica/comunicacion/publicaciones/dotacion-y-condiciones-de-trabajo-de-enfermeria-en-el-uruguay [Consulta: 5 enero 2018].

17. Servicio Madrileño de la Salud. Enfermería en Atención Primaria. Madrid: Biblioteca Lascasas, 2005:57p. Disponible en: $\quad \mathrm{http} / / / w w w . i n d e x-f . c o m / l a s c a s a s / d o c u m e n-$ tos/1c0079.php [Consulta: 24 setiembre 2018].

18. Organización Panamericana de la Salud-Organización Mundial de la Salud. La cumbre de enfermería de práctica avanzada: desarrollando competencias de enfermería de práctica avanzada en Latinoamérica para contribuir a la salud universal. (Ann Arbor, Michigan, 7-9 de abril, 2016) Michigan: PHAO, 2016.

19. Uruguay. Ley $\mathrm{N}^{\circ} 18815$. Regulación del Ejercicio de la Profesión Universitaria de Enfermería y de Auxiliar de Enfermería. Montevideo, 30 de setiembre de 2011). Disponible en: https://www.impo.com.uy/bases/leyes/18815-2011 [Consulta: 5 enero 2018].

20. Decreto 354/014. Reglamentación de la Ley N 18815 . Montevideo, 4 de diciembre de 2014. Disponible en: https://www.impo.com.uy/bases/decretos/354-2014.[Consulta: 5 enero 2018].

21. Uruguay. Universidad de la República. Enfermería apuesta a formar más profesionales y a diversificar las tareas. (Entrevista a la Decana de Facultad de Enfermería Lic. Mercedes Pérez, publicado el lunes 31 de agosto de 2015. Disponible en: http://www.universidad.edu.uy/prensa/renderItem/itemId/37790 [Consulta: 15 setiembre 2018].

22. Uruguay. Universidad de la República. Facultad de Enfermería. Dpto. de Enfermería Comunitaria. Aportes desde la formación del Licenciado en Enfermería en el área comunitaria: una contribución al SNIS. 2017. Disponible en: http://www.msp.gub.uy/sites/default/files/archivos_adjuntos/1_Aporte\%20desde\%201a\%20formaci $\%$ C3\%B3n $\% 20$ del $\% 20$ licenciado $\% 20$ en $\% 20$ enfermer\%C3\%ADa $\% 20$ en $\% 20$ el $\% 20 \% \mathrm{C} 3 \%$ A1 rea $\% 20$ comunitaria.pdf [Consulta: 3 setiembre 2017].

23. Uruguay. Universidad de la República. Facultad de Enfermería de la Udelar obtiene acreditación regional.(Publicado el 16 de julio de 2018). Disponible en: http://www.universidad.edu.uy/prensa/renderItem/itemId/42308.[Consulta: 15 setiembre 2018].

24. Uruguay. Ministerio de Salud Pública. Situación de los recursos humanos en salud del Uruguay. BE 01/14. Bol Estad

\section{Contribución de autores}

Las autoras participaron en igual medida en la elaboración del artículo

Stela Harispe, https://orcid.org/0000-0001-5790-6688

Fiorella Cavalleri, https://orcid.org/0000-0002-0028-3544

Ima León, https://orcid.org/0000-0001-5854-6362 
2014. Disponible en: http://www.msp.gub.uy/publicaci\%C3\%B3n/bolet $\%$ C3\%ADn-estad\%C3\%ADstico-2014 [Consulta: 12 agosto 2018].

25. Uruguay. Administración de los Servicios de Salud del Estado. Primer Curso de Consulta de Enfermería desde un abordaje Familiar y Comunitario. (Montevideo, 3 agosto 2018). Disponible en: http://www.asse.com.uy/contenido/Evento-Apertura-Primer-Curso-Consulta-de-Enfermeria-Desde-un-Abordaje-Familiar-y-Comunitario-10834 [Consulta: 24 setiembre 2018]. 